\title{
SUBLIMINAL LASER AS ADDITIONAL THERAPY TO REDUCE INTRAVITREAL INJECTION NUMBER FOR THE PATIENTS WITH MACULAR DISEASES
}

\author{
Liene Muceniece *, **, Dace Markevica ***, **** \\ * Riga Stradins University, Riga, Latvia \\ ** Riga Health Centre, Riga, Latvia \\ *** Pauls Stradins Clinical University Hospital, Riga, Latvia \\ **** Eye Health Centre, Riga, Latvia
}

\begin{abstract}
Summary
Introduction. Intravitreal injections have become one of the most performed surgical procedures in ophthalmology. There is a need to look for an effective alternative therapy for patients with anti-VEGF complications and systemic disease contraindications. Aim of the study. To evaluate Subliminal laser effectiveness on reducing macular disease edema.

Materials and methods. Subliminal laser treatment was done three times with three month intervals for patients with macular edema due to retinal diseases. On 1st, 3rd, 6th and 9th month visit the best corrected visual acuity, intraocular pressure, macular thickness and macular volume were registered.

Results. Mean visual acuity before treatment was $0,52(S D=0,31)$ that changed to 0,56 after the 9 month treatment $(p>0,05)$. Mean central macular thickness from $326,12 \mu \mathrm{m}$ reduced to $308,15(p=0,76)$. Without significant difference, inner cycle volume changed from 1353,80 mm3 to 1297,57 mm3 ( $p=0,81)$ and outer cycle volume from 1179,4 mm3 to 1112,56 mm3 ( $p=0,51)$.

Conclusions. Subliminal laser controls macular edema level and could be a solution for patients who cannot afford or are emotionally distressed by monthly intravitreal injections. This treatment method can be used as additional therapy to reduce the number of intravitreal injections.
\end{abstract}

Keywords: Subliminal laser, Macular disease, Intravitreal injections

\section{INTRODUCTION}

In the past decade, intravitreal injections have become one of the most performed surgical procedures in ophthalmology (1). It is a fast manipulation that can cause serious ophthalmological complications as well psychological discomfort for patients due to repeated trauma to the eye. Due to comorbidities, anti-vascular endothelial growth factor (anti-VEGF) treatment may be accompanied with an increased risk of complications or limited possibility for its usage at all.

Infectious endophthalmitis are reported to be $0,019 \%$ to $1,6 \%$ of intravitreal injection patients (2). Intraocular inflammation in research has been reported to be 1,4\%$2,9 \%$ even with well-known aseptic preparation before, during, and after injection (2). Sterile instrument use, face mask and sterile gloves are required during the manipulation for each injection. Post-injection topical antibiotic use is still a controversial practice. Infectious endophthalmitis cases are independent of geographical location, injection settings, and different anti-VEGF agents. Still, patients with active external ocular infections or eyelid abnormalities are more at a risk of endophthalmitis (2). If possible, these ocular problems should be solved before starting injection treatment. If intraocular inflammation or infectious endophthalmitis happens, it results into additional long and complex treatment plans.

Low incidence of rhegmatogenous retinal detachment $(<0,67 \%)$ can be explained by correct injection technique (2). There is still no clear mechanism for intraocular pressure elevation after injection. It includes theories of inflammatory mechanism, impaired outflow owing to protein aggregates, and damage to outflow pathways (2). Around 10\% of injections result in subconjunctival hemorrhage. Even if it is just a cosmetic defect, it causes a mild discomfort for a patient.

High levels of anxiety have been reported for $67 \%$ of patients for their first injection. This slowly decreases after the subsequent injections (3). Most anxieties are the caused by needle entry in the eye, but in addition time spent in the waiting room and traveling to manipulation is a reason to get overwhelmed. Because of low visual acuity, people are usually accompanied by relatives or friends to intravitreal injection manipulation. The need of emotional support affects their subjective pain and anxiety (4). As recommended, intravitreal injection therapy should be done monthly with prognostic high expenses and possible complications (5).

Subliminal laser has begun to be used in diabetic macular edema and central serous chorioretinopathy treatments. This method is well tolerated by patients because it is painless and should be done every 3 months (6). In the micropulse mode, applied energy is divided into short pulses (7). Laser emission is absorbed by the xanthophyll pigment and doesn't cause any laser-induced retinal damage $(5,8)$. This treatment 
activates biochemical processes but does not cause damage to retinal layers.

\section{AIM OF THE STUDY}

The aim of our study was to evaluate subliminal laser effectiveness on reducing macular disease edema.

\section{MATERIALS AND METHODS}

A retrospective study was designed in the Eye Health Centre (Riga, Latvia). Treatment with a Yellow Subliminal laser $(577 \mathrm{~nm})$ was conducted for 25 eyes from February, 2017 until April, 2018. It was used for patients with diabetic macular edema, retinal edema after retinal vein thrombosis, central serous chorioretinopathy, and age related macular degeneration. Only for patients with macular edema, less than $600 \mu \mathrm{m}$ were included in this research. Laser settings were applied as 5\% duty cycle, $160 \mu \mathrm{m}$ spot size and $200 \mathrm{~ms}$ exposure time.

In total, laser treatment was done three times with three month intervals. For each patient, the following were registered before laser treatment and on the 3rd, 6th and 9th month visit: best corrected visual acuity (Snellen chart), intraocular pressure (ICare), macular thickness and macular volume. Retinal thickness and macular volume were registered with spectral domainoptical coherence tomography (Topcon). Before laser treatment, a topical anesthetic was applied. After laser treatment, an antibacterial drop is inserted into the lower eyelid sulcus.

Macular volume was analyzed in 3 sectors: central macula $-1 \mathrm{~mm}$ area around fovea; inner macula -3 $\mathrm{mm}$ diameter around fovea; and outer macula $-6 \mathrm{~mm}$ diameter (Fig. 1). Inner and outer macular volumes were calculated for all four sectors in this diameter. Statistical analysis was done with Wilcoxon and Spearman's correlation tests and descriptive statistics by IBM SPSS Statistics 22.0

\section{RESULTS}

Mean visual acuity before treatment was 0,52 $(S D=0,31)$ that changed to $0,56(0,31)$ after the treatment, without any significant difference $(p>0,05)$. Visual acuity drop on the 6 th month visit to $0,48(0,28)$ is significant $(p=0,023)$, but it returned to the previous level on the 9 th month visit. There were no significant ocular tension changes during the treatment $(p=0,72)$. Before starting treatment, mean ocular tension was $14,96(\mathrm{SD}=2,74) \mathrm{mmHg}$, which increased to 15,62 $(3,79) \mathrm{mmHg}$ on the 6 th month visit, but dropped to $13,11(3,21) \mathrm{mmHg}$ at the end of treatment.

Mean central macular thickness before treatment was $326,12 \mu \mathrm{m}$, which did not significantly change after 9 months of treatment to $308,15(\mathrm{p}=0,76)$. Inner cycle volume before laser treatment was $1353,80 \mathrm{~mm}^{3}$ and it reduced to $1297,57 \mathrm{~mm}^{3}$ at 9 th month visit without significant difference $(p=0,81)$. Outer cycle volume, as well, did not significantly change from $1179,4 \mathrm{~mm}^{3}$ to $1112,56 \mathrm{~mm}^{3}(\mathrm{p}=0,51)$.

There is a strong $(\rho=0,84)$ and significant $(p=0,004)$ correlation with central macular thickness before and after treatment (Fig. 2). There were similar findings with inner cycle volume, where there was a strong correlation $(\rho=0,84)$ before and after treatment that was significant $(p=0,01)$. Outer cycle volume shows a strong correlation before treatment to the 3rd month visit $(\rho=0,88, p=0,001)$ and to the 9 th month visit $(\rho=0,88, p=0,002)$ (Fig. 3).

\section{DISCUSSION}

In our research it is shown that subliminal laser treatment does not have a high positive effect on resolving retinal edema; still it controls edema levels and doesn't allow it to progress. Ying $\mathrm{Wu}$ et al have shown in their research that subliminal laser BCVA improvement and central macular thickness is not statistically changing with a subliminal laser monotherapy treatment, but still is more effective than conventional laser treatment for diabetic macular edema (5). In addition, Parodi et al reported that after subliminal laser treatment there is only limited functional improvement for macular edema in branch retinal vein occlusion (8). Subliminal lasers could be a helpful method to reduce the intravitreal injection number for a person, but will not be effective monotherapy for retinal edema. It could be a solution for patients that can't afford to do monthly intravitreal injections to keep macular edema controlled. Ying $\mathrm{Wu}$ et al discussed that first-line therapy for diabetic macular edema should begin with subliminal laser for patients in low or intermediate resource settings (5).

Subliminal laser is more effective on smaller edema and it is suggested to be used for edema that is not more than $400 \mu \mathrm{m}$. In our research we limited it to $600 \mu \mathrm{m}$. Even with no significant effect, numerical central macular thickness after treatment is reduced even for a patients with edema more than $400 \mu \mathrm{m}$. This effect is not only found in macular central thickness, but is noticeable for inner and outer circle volume. Subliminal laser use has been reported as a good combination therapy for refractory macular edema (6). It leads one to think that subliminal laser could be considered as an alternative treatment method for a small edema or small edema with anti-VEGF treatment. With previous research, this method should be considered for certain types of macular edemas.

There is a patient group who cannot receive anti-VEGF intravitreal injections due to systemic conditions. There are reports of increased mortality, cardiac ischemic and thromboembolic events in patients with cancer who received bevacizumab treatment. Even with this small rate, there is a greater risk for infections and gastrointestinal disorders (9). In these patients, authors think subliminal laser could be a way to control edema. Still, it is understandable that it will not give the proper reduction of retinal edema.

\section{CONCLUSIONS}

Subliminal laser treatment for patients with diabetic macular edema, retinal edema after retinal vein 
thrombosis, central serous chorioretinopathy, and age related macular degeneration could be used as additional therapy to first-line treatment with anti-VEGF. This treatment method controls macular edema levels and could be a solution for patients who cannot afford or have emotional distress of monthly intravitreal injections.

\section{Conflict of interest: None}

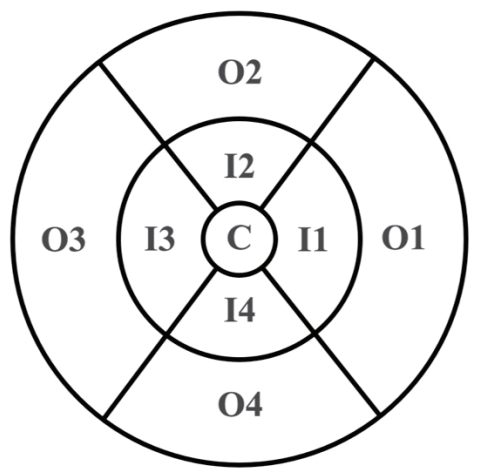

Fig 1. Sectoral map in alphanumeric form for macular volume analysis, where $\mathrm{C}$ - central, I inner and $\mathrm{O}$ - outer macula.

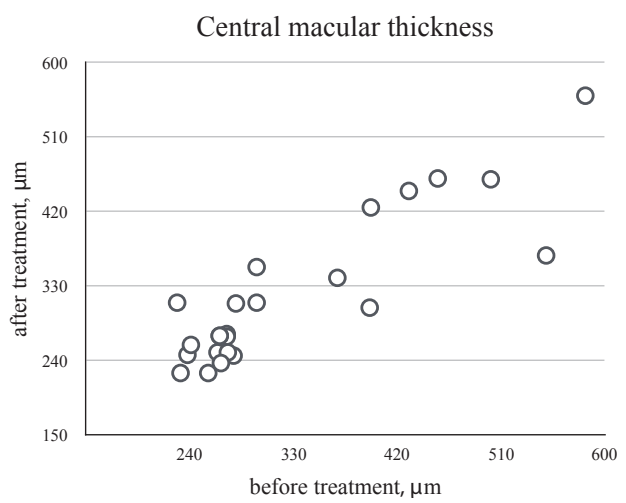

Fig 2. Central macular thickness change before and after 9 months of the treatment with the subliminal laser.

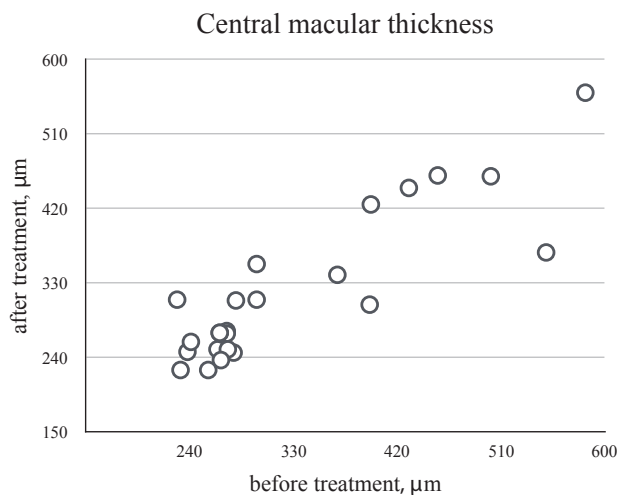

Fig 3. Macular outer circle volume change before the treatment and after 3 months and 9 months of the treatment.

\section{REFERENCES}

1. Lai T, Liu S, Das S, Lam D. Intravitreal Injections Technique and Safety // Asia Pac J Ophthalmol, 2015; 4:321-328

2. Falavarjani KG, Nguyen QD. Adverse events and complications associated with intravitreal injection of antiVEGF agents: a review of literature // Eye, 2013, 27(7):787-794

3. Spooner KL, Guinan G, Koller S, Hong T, Chang AA. Burden of Treatment Among Patients Undergoing Intravitreal Injections for Diabetic Oedema in Australia // Diabetes Metab Syndr Obes, 2019; 12:1931-1921

4. Sii S, Aspinall P, Borooah S, Dhillon B. Exploring factors predicting changes in patients' expectations and psychosocial issues during the course of treatment with intravitreal injections for wet age-related macular degeneration // Eye, 2017, 32(4):673-678

5. Wu Y, Ai P, Ai Z, Xu G. Subthreshold diode micropulse laser versus conventional laser photocoagulation monotherapy or combined with antiVEGF therapy for diabetic macular edema: A Bayesian network meta-analysis // Biomed Pharmacother, 2018, (97):293-299

6. AkhlaghiM, Dehghani A etal.Effects of subthreshold diode micropulse laser photocoagulation on treating patients with refractory diabetic macular edema // J Curr Ophthalmology, 2018, 31(2): $157-160$

7. Gonzales-Saldivar G, Rojas-Juarez S, EspinosaSoto I et al. Single-Spot Yellow Laser Versus Conventional Green Laser on Panretinal Photocoagulation: Patient PAin Scores and Preferences // Ophthalmic Surg Lasers Imaging Retina, 2017, 48(11):902-905

8. Parodi M B, Iacono P, Bandello F. Subthreshold grid laser versus intravitreal bevacizumab as a second line therapy for macular edema in branch retinal vein occlusion recurring after conventional grid laser treatment // Graefes Arch Clin Exp Ophthalmol, 2015, (10):1647-1651

9. Lim LS, Cheung CMG, Mitchell P, Wong TY. Emerging evidence concerning systemic safety of anti-VEGF agents - should ophthalmologists be concerned? // Am J Ophthalmol, 2011 152(3): 329-33

\section{Address:}

Liene Muceniece

Pauls Stradins Clinical University Hospital

Pilsoņu iela 13, Rīga, LV-1002

Email: liene.muceniece@gmail.com 